\title{
A new modified animal model of myosin-induced experimental autoimmune myositis enhanced by defibrase
}

\author{
Luo Wen-Jing ${ }^{1,2}$, Pu Chuan-Qiang ${ }^{1}$, Li Hong-Hua ${ }^{2}$, Lu Xiang-Hui ${ }^{1}$, Liu Jie-Xiao ${ }^{1}$
}

\begin{abstract}
${ }^{1}$ Department of Neurology, Chinese PLA General Hospital, Beijing, China 2Department of Neurology, Chinese PLA Wuhan General Hospital of Guangzhou Military Command, Wuhan, China
\end{abstract}

Submitted: 21 March 2014

Accepted: 27 June 2014

Arch Med Sci 2015; 11, 6: 1272-1278

DOI: 10.5114 /aoms.2015.52883

Copyright @ 2015 Termedia \& Banach

\section{Abstract}

Introduction: We investigated the effect of defibrase (a proteolytic enzyme extraction of Agkistrodon halys venom) on experimental autoimmune myositis (EAM) in guinea pigs and explored the option of using a modified pig model of EAM to enhance the study of this disease.

Material and methods: Guinea pigs were divided into 3 groups: group A (control group) was immunized with complete Freund adjuvant (CFA), then received 6 injections of saline weekly; group B (EAM group) was immunized with partially purified rabbit myosin emulsified with CFA, then received an injection of saline; group C (EAM + defibrase group) was immunized with purified rabbit myosin emulsified with CFA, then received an injection of defibrase. The animals were observed for their general health condition and the body weight was measured daily. Plasma levels of fibrinogen and creatine kinase (CK) were determined. Muscle tissues were examined histologically. Results: After immunizations for 6 weeks, incidence of EAM in groups A, $\mathrm{B}$ and $\mathrm{C}$ was $0(0 / 7), 83.3 \%(10 / 12)$ and $100 \%(15 / 15)$, respectively. Guinea pigs with EAM presented angeitis symptoms of muscle weakness. Histological analysis revealed a significant difference. Muscles with EAM had scattered or diffuse inflammatory manifestations, which are also common pathological features of human idiopathic polymyositis (IPM). Defibrase-treated animals displayed extensive inflammation and fiber necrosis compared with the EAM group (histological score: $2.80 \pm 1.15$ vs. $1.88 \pm 1.32$, $p<0.05)$. Severity of inflammation of group B was mainly mild to moderate; $16.7 \%(2 / 12)$ of animals developed severe inflammation. Incidence of severe inflammation with a score up to 4 in group $C$ was $40 \%(6 / 15)$.

Conclusions: Defibrase can exacerbate myosin-induced EAM; thus a new modified model was generated.

Key words: experimental autoimmune myositis, guinea pigs, idiopathic polymyositis, defibrase, fibrinogen.

\section{Introduction}

Idiopathic polymyositis (IPM) is an acquired autoimmune inflammatory myopathy which affects skeletal muscles [1], and is characterized for having moderate to severe muscle weakness and histological skeletal muscle inflammation. However, the etiology of IPM has not been well elucidated. Experimental autoimmune myositis (EAM) is an animal

\author{
Corresponding author: \\ Pu Chuan-Qiang \\ Department of Neurology \\ Chinese PLA General Hospital \\ No. 28 Fuxing Road \\ Beijing 100853, China \\ Phone: 86-10-6693 6391 \\ E-mail: \\ chuanqiangpu67@163.com
}


model induced with similar pathological changes to that of human IPM. Reports demonstrated that purified myosin only generated mild to moderate myositis in guinea pigs, which limited further research on severe IPM [2, 3]. Thus we attempted to create a new model in guinea pigs with severe inflammation and muscular fiber necrosis.

Fibrinogen is a glycoprotein converted to insoluble fibrin by activated thrombin [4], which mediates multiple physiological responses including platelet aggregation, clot formation, cell adhesion and wound healing [5-7]. Fibrinogen also represents one of the major acute-phase proteins, and its biosynthesis increases significantly in inflammation and stress $[7,8]$. Through direct binding to specific receptors on inflammatory or tumor cells, fibrinogen leads to synthesis of proinflammatory cytokines [9]. Compelling studies demonstrated that the plasma level of fibrinogen increased when cardiovascular disease, bacterial infection, rheumatoid arthritis, glomerular nephritis and experimental allergic encephalomyelitis (EAE) occurred. This evidence indicates that fibrinogen is a biomarker of inflammation and tumor [10-12].

We hypothesized that fibrinogen is increased in plasma of IPM and reducing the fibrinogen level could have a therapeutic effect on IPM. Defibrase, a drug used for degradation of fibrinogen, is commonly used for therapies of acute ischemic cerebrovascular disease, thromboangiitis obliterans, deep phlebitis and sudden deafness. Few reports have mentioned its effect on IPM. In this study, we elucidated the effect of defibrase on EAM.

\section{Material and methods}

\section{Animals}

The following animal protocols were approved by the Institutional Animal Care and Use Committee at the Chinese PLA General Hospital Medical Center.

Undercoat female guinea pigs were purchased from Chinese PLA General Hospital Animal Center (Beijing, China) and bred in our animal house at $18-20^{\circ} \mathrm{C}$. Used guinea pigs were $8-10$ weeks old, about 250-300 g. To determine the effects of myosin and defibrase on EAM, the animals were divided into 3 groups randomly: group $A$ (control group, $n=7$ ); group B (EAM group, $n=12$ ) and group C $(E A M+$ defibrase group, $n=15)$.

\section{Experimental autoimmune myositis induction and defibrase administration}

For guinea pigs in group $A$, injection of $0.01 \mathrm{M}$ PBS + complete Freund adjuvant (CFA) was performed on a weekly basis. After 6 immunizations, saline was administrated 4 times.

Group $B$ animals received an injection of myosin in 0.01 M PBS + CFA on a weekly basis. Af- ter 6 immunizations, saline was administrated 4 times.

For group C, injection of myosin in $0.01 \mathrm{M}$ PBS + CFA on a weekly basis was performed. After 6 immunizations, defibrase was administrated 4 times.

Guinea pigs were immunized by subcutaneous injections of $0.25 \mathrm{ml}$ purified rabbit myosin (10.8 mg/ml (Biuret), 0.75 units/mg protein, $\mathrm{pH}$ 6.8, Sigma-Aldrich, St. Louis, MO, USA) emulsified with an equal volume of conventional CFA containing $1 \mathrm{mg} / \mathrm{ml}$ M. tuberculosis (Sigma-Aldrich, St. Louis, MO, USA) in multiple sites of the back on a weekly basis 6 times. At the time of immunization, animals received an intraperitoneal injection of $0.3 \mathrm{ml}$ of pertussis bacterium solution $\left(2 \times 10^{9} / \mathrm{ml}\right.$ pertussis bacterium, Tiantan Bio Ltd., Beijing, CN).

\section{Sample collection}

After 1 day following the last immunization, animals in groups $A$ and $B$ were injected with saline intraperitoneally every other day 4 times. Group C guinea pigs received defibrase (a proteolytic enzyme extraction of Agkistrodon halys venom, $20 \mathrm{U} /$ kg, Saisheng Pharmacy Ltd, Beijing, CN) injections with the same protocol. One day after the last drug administration, animals were anesthetized with $10 \%$ chloral hydrate intraperitoneal injection. Blood was drawn through abdominal aorta puncture, and collected in sodium citrate tubes and clot activator tubes. The samples were set at room temperature (RT) for $1 \mathrm{~h}$ and centrifuged at $1500 \mathrm{~g}$. The supernatant liquid was stored at $-20^{\circ} \mathrm{C}$ for detection of creatine kinase (CK) levels. Muscles of proximal parts of extremities were ectomized and embedded in tragacanth gum. Then the tissues were snap frozen in isopentane pre-chilled with liquid nitrogen and stored at $-80^{\circ} \mathrm{C}$ for histological analysis.

\section{Animal phenotypes score evaluation}

The animals were weighed daily, and their appearance and behavioral changes were observed. From the second immunization, every animal was scored by their symptoms with the following criteria: 0 , no obvious muscle weakness; 1 , mild weakness, such as biting weakness and (or) hoarse voice; 2 , moderate weakness, such as bent back at static state, dropped head and staggering gait; 3, severe weakness, muscular atrophy, no biting, even dyspnea and nearly dead. The animals with intermediate symptoms were graded as 0.5, 1.5 and 2.5 .

\section{Laboratory examination}

Plasma levels of fibrinogen were determined quantitatively by the clotting method of Clauss 
with an STA-fibrinogen kit (Stago, Paris, FRA). The test principle is that in the presence of an excess of thrombin, the clotting time of diluted plasma has a direct bearing on the level of plasma fibrinogen. CK activities of serum were detected by the UV-test method with the cobas-creatine kinase kit (Roche/Hitachi, Mannheim, GER). Detailed operating procedures followed the manufacturer's instructions.

\section{Histological analysis}

Histological analysis was performed by hematoxylin and eosin-stained sections. Briefly, muscle degeneration was characterized by sarcoplasmic swelling, pallor, and vacuolization. Necrotic myofibers showed sarcoplasmic hypereosinophilia with loss of cross-striations, fragmentation, nuclear pyknosis, karyorrhexis, and karyolysis. The inflammatory profile consisted of inflammatory cell (macrophages and lymphocytes) infiltration.

Regenerative changes include satellite cell activation/migration, myofibers with basophilic cytoplasm, nuclear internalization, and large nuclei with prominent nucleoli. The histological severity of inflammation in each muscle block was graded into 4 categories [13]: grade 1 , involvement of a single muscle fiber or less than 5 muscle fibers; grade 2, a lesion involving 5-30 muscle fibers; grade 3 , a lesion involving a muscle fasciculus; grade 4 , a diffuse extensive lesion. When multiple lesions with the same grade were found in a single muscle block, 0.5 point was added to the score.

\section{Statistical analysis}

The results are expressed as means \pm SD. Statistical differences between groups were compared by Student's $t$ test or one-way analysis of variance (ANOVA) test. Value of $p<0.05$ was considered as statistically significant. Statistical analysis was performed using SPSS13.0 software (IBM, IL, USA).

\section{Results}

\section{Symptoms and laboratory results}

The evaluated scores of muscle phenotypes, blood profile and histological analysis in different groups are shown in Table I. From the second immunization, subcutaneous nodules of guinea pigs began to form at the local sites of injection in groups B and C. Skin changes included swelling and ulcers of the back skin, hair loss, and dirty and dull hair. Animals in group A had no obvious weakness and showed normal mobility and a steady increase in weight during the experimental period. From the second week, immunized animals of groups $B$ and $C$ showed different degrees of muscle weakness and weight loss. Myosin-immunized guinea pigs displayed typical symptoms of muscle pain including trailing hind limbs, reduced locomotor activity, dropped head and staggering gait. One immunized animal developed severe weakness 1 day after administration of defibrase with inability to turn over, dyspnea and was nearly dead. The animal was sacrificed ahead of schedule.

However, no significant difference appeared in evaluated scores between groups B and C (1.38 \pm 0.68 vs. $1.57 \pm 0.7, p>0.05$ ).

As we can see from Table I, there was no significant difference in CK $(F=0.48, p=0.63)$ and fibrinogen level between the 3 groups $(p>0.05)$. In order to explore the relationship between CK levels and phenotype grading, Pearson correlation analysis was performed, but no correlation $(r=-0.238$, $p=0.255)$ was found. This pattern was inconsistent with that of human IPM, in which CK level usually was a sensitive biomarker of disease activity.

\section{Defibrase induces intensive inflammation and necrosis}

Histological analysis results (Table I) revealed no existence of obvious necrosis or inflammation in group A animals. No animal showed any dys-

Table I. CK activity, fibrinogen level, incidence of clinical manifestation, evaluated score, incidence and severity of EAM in different groups

\begin{tabular}{|c|c|c|c|}
\hline Parameter & Group A & Group B & Group C \\
\hline \multicolumn{4}{|l|}{ Blood profile: } \\
\hline Median CK activity [U/I] & 1414.25 & 1423.50 & 1902.70 \\
\hline Mean fibrinogen level [g/l] & $1.90 \pm 1.40$ & $1.56 \pm 0.27$ & $1.51 \pm 0.56$ \\
\hline \multicolumn{4}{|l|}{ Muscle phenotypes: } \\
\hline Incidence of clinical symptoms (\%) & 0 & $12 / 12(100.0)^{*}$ & $15 / 15(100.0)^{*}$ \\
\hline Evaluated score & 0 & $1.38 \pm 0.68^{\star}$ & $1.57 \pm 0.70^{*}$ \\
\hline \multicolumn{4}{|l|}{ Histological analysis: } \\
\hline Incidence of EAM (\%) & $0 / 7(0)$ & $10 / 12(83.3)^{*}$ & $15 / 15(100.0)^{*}$ \\
\hline Histological score & 0 & $1.88 \pm 1.32^{*}$ & $2.80 \pm 1.15^{\text {*\# }}$ \\
\hline
\end{tabular}


trophy, degeneration or necrosis of muscle fiber, or infiltration of inflammatory cells (Figure $1 \mathrm{~A}$ ). However, the majority of animals in group $B$ had intensive inflammation and necrosis, and 83.3\% of animals suffered EAM confirmed by histological examination. Muscles of EAM displayed mild to severe inflammatory features of degeneration, necrosis and phagocytosis of fibers with infiltration of mononuclear cells in the endomysium and epimysium (Figures 1 B, C). For group C, defibrase induced intensive inflammation and necrosis. The muscle pathology data in 3 groups are collected in Table II. All the animals displayed histological features of myositis. The histological score in group $C$ was the highest among the 3 groups. More se-
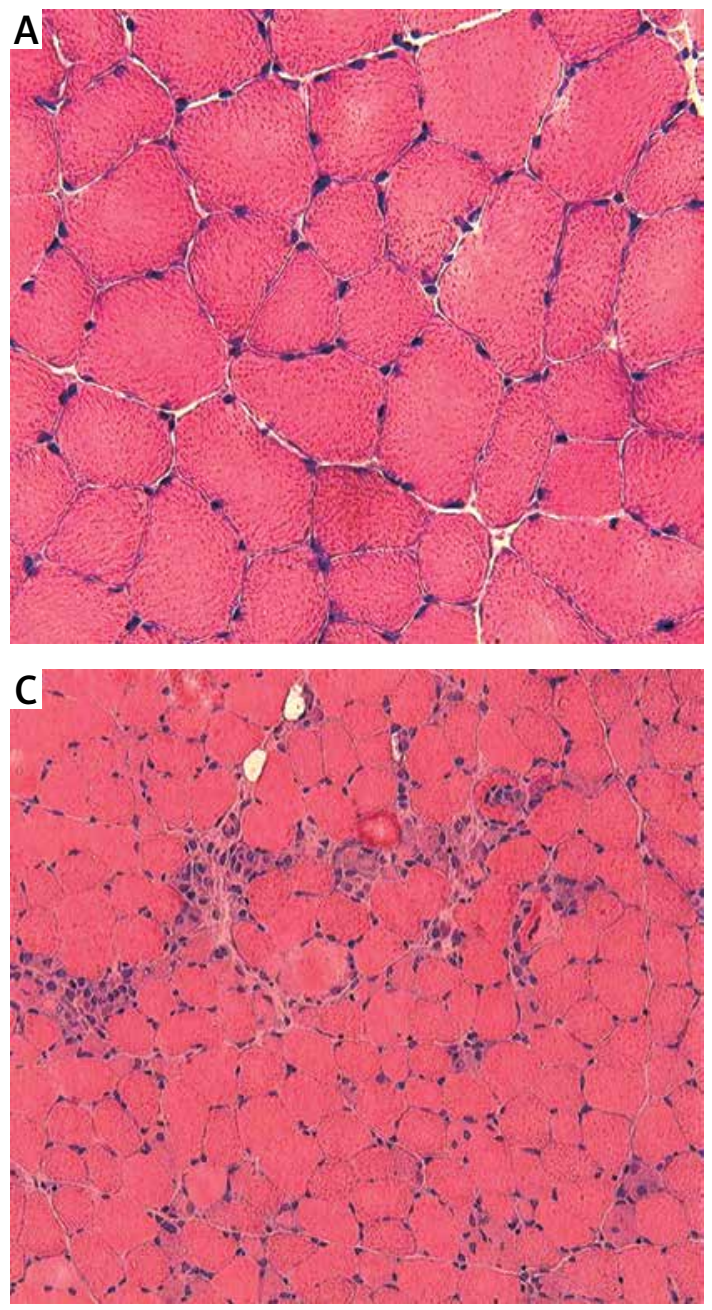

vere inflammatory damage was observed in this group (and) 40\% (6/15) of animals developed extensive lesions with a score up to 4 . We observed that many fibers were necrotic, accompanied with infiltrate of numerous mononuclear cells, macrophages and plasmocytes (Figures $2 \mathrm{~A}-\mathrm{C}$ ). However, the majority of animals in group $B(8 / 12,66.7 \%)$ displayed mild to moderate inflammation with a score of $1-2.5$ and only $16.7 \%(2 / 12)$ of animals had intensive inflammation (Table II).

\section{Discussion}

Previous studies had demonstrated that partially purified skeletal myosin could induce severe EAM in rats or mice $[13,14]$. Other antigens used

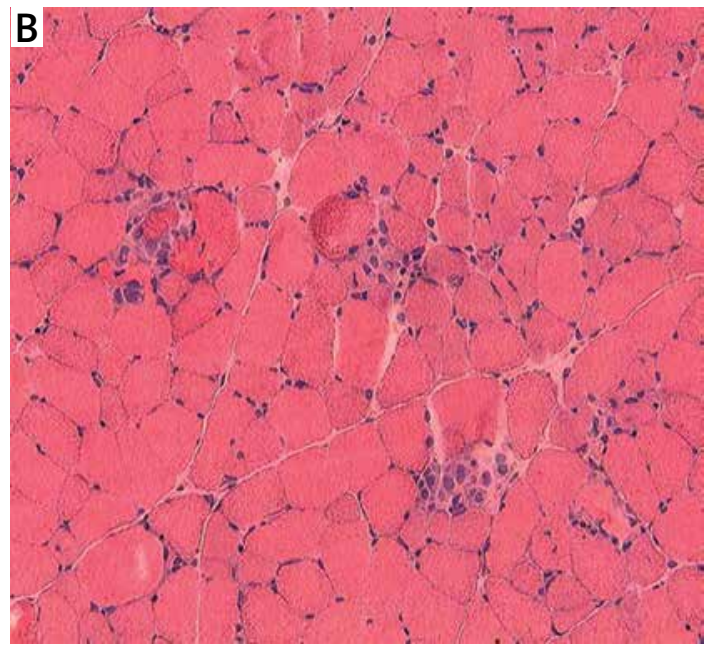

Figure 1. HE stain result of muscle in 3 groups (100x). A - Group A (normal control), morphology and structure of muscle fibers are normal, no infiltration of inflammatory cells could be observed. B - Group B (EAM), initial inflammation involving a single fiber with infiltration of mononuclear inflammatory cells, particle muscle fiber was degenerated, necrosis could be observed with surrounding infiltrating mononuclear cells. C - Group B, a number of fibers were degenerated and necrotic, surrounded by mononuclear cells

Table II. Muscle pathology data in 3 groups

\begin{tabular}{|lcccccc|}
\hline Group & $\begin{array}{c}\text { Total } \\
\text { number }\end{array}$ & $\begin{array}{c}\text { Pathology } \\
\text { score }=\mathbf{0}(\mathrm{N})\end{array}$ & $\begin{array}{c}\text { Pathology } \\
\text { score }=\mathbf{1}(\mathrm{N})\end{array}$ & $\begin{array}{c}\text { Pathology } \\
\text { score }=2(\mathrm{~N})\end{array}$ & $\begin{array}{c}\text { Pathology } \\
\text { score }=\mathbf{3}(\mathrm{N})\end{array}$ & $\begin{array}{c}\text { Pathology } \\
\text { score }=\mathbf{4}(\mathrm{N})\end{array}$ \\
\hline A (control) & 7 & 7 & 0 & 0 & 0 & 0 \\
\hline B (EAM) & 12 & 2 & 4 & 4 & 0 & 2 \\
\hline C (EAM + defibrase) & 15 & 0 & 5 & 3 & 1 & 6 \\
\hline
\end{tabular}



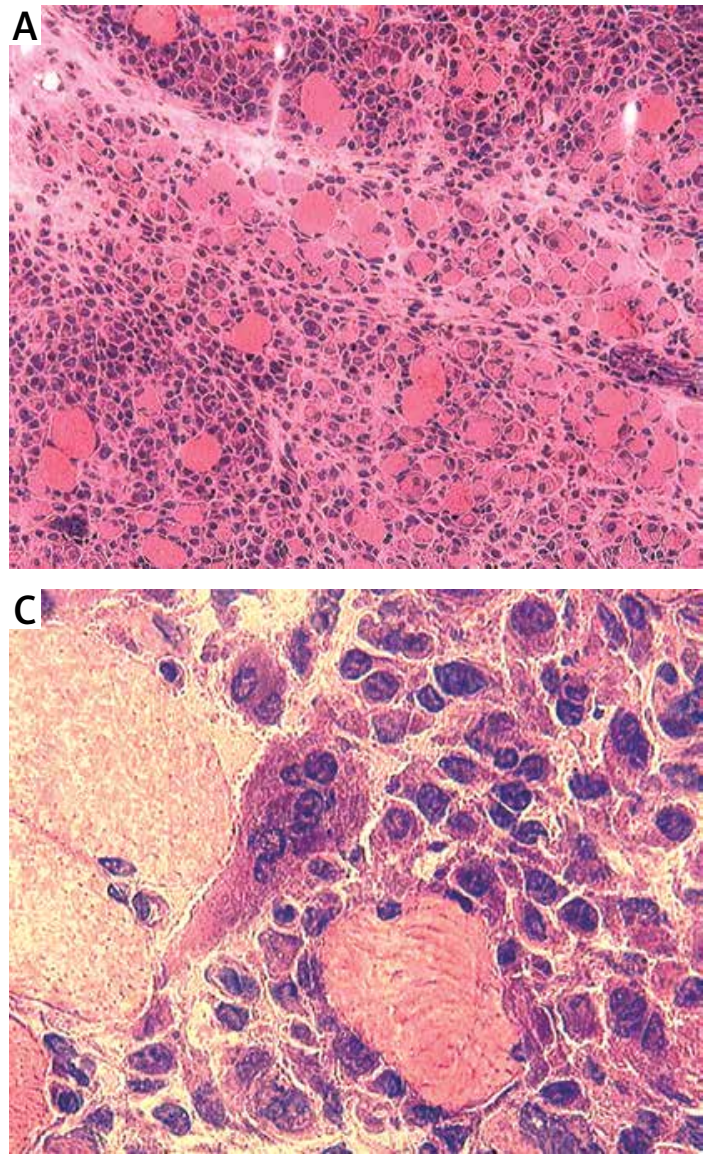

in EAM induction included whole rabbit muscle extract, purified myosin, C-protein and laminin [1416]. In the present study, we successfully induced EAM in guinea pigs with purified rabbit myosin. Pertussis bacterium solution (Bordetella pertussis (ATCC 9797, $4.3 \times 108 / 20 \mathrm{ml}$ )) was added as an ancillary antigen. This EAM animal model shared some characteristics of human IPM in phenotype symptoms. EAM animals showed muscle weakness including biting weakness, hoarse voice, decreasing mobility, trailing hind limbs, dropped head, staggering gait and dyspnea.

After administration of defibrase, the animal showed severe weakness with no ability to turn over and was nearly dead. Histological examination revealed diffuse fiber necrosis, phagocytosis and infiltration of numerous mononuclear cells. Although EAM and defibrase treated animals showed various degrees of weakness and weight loss, there was no significant difference in the phenotype score between groups B and C. Some animals only showed mild to moderate weakness, but their histological analysis demonstrated multiple intensive inflammatory lesions. We speculate that the probable reason for this discrepancy between their symptoms and pathological manifestations was inaccurate measurement of muscle strength in animals.

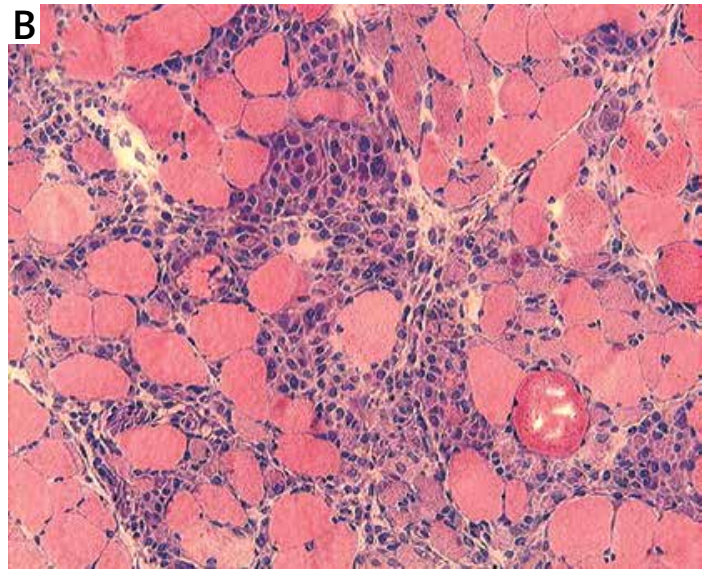

Figure 2. HE stain results of muscle in group $C$ (EAM + defibrase group). A - Extensive inflammatory lesions could be observed in the muscle, 100x. B - The inflammation was severe, degeneration, necrosis, muscle fiber dissolution, endomysium and perimysium lymphocytes, and epimysial inflammation could be observed, 100x. C - Besides a large number of mononuclear cells, a polykaryocyte could also be found, $200 x$

However, we found that purified myosin only induced mild to moderate EAM, which could not result in severe enough inflammation. Against our expectations, the defibrase-treated group showed more diffuse inflammation and necrosis. Histological scores of the defibrase-treated group were higher than those of the solely myosin induced group. Histological analysis revealed diffuse inflammatory infiltration existing in the endomysium and perimysium, extensive phagocytosis and necrosis.

Fibrinogen is a soluble $340 \mathrm{kDa}$ dimeric glycoprotein synthesized by liver and has diverse biological activities. The most well-known function of fibinogen is blood coagulation. After initiation of the coagulation cascade, the serine protease thrombin cleaves peptides from the $\mathrm{N}$-terminal regions of the $A \alpha$ and $B \beta$ chains. The cleavage of these peptides exposes polymerization sites and initiates the formation of a polymer consisting of non-covalently linked protofibrils, which form an insoluble fibrin clot through binding activated coagulation factor XIII. During this process, a variety of pathological processes are involved including inflammation and immunity through its integrin and non-integrin receptors. Previous studies have demonstrated that fibrinogen deposited in tissues plays an important role in inflammatory processes including bacterial infection, glomerulonephritis and rheumatoid 
arthritis [17-19]. It is often indicated that as an acute-phase protein, fibrin(ogen) deposition had also been found in several nervous system diseases including stroke [12], Alzheimer's disease [20], peripheral nerve injury [21] and multiple sclerosis [22]. Endothelial cells are activated, which results in leakage of fibrinogen from blood vessels. Fibrinogen is subsequently transformed into fibrin, which regulate migration and adhesion through interactions between extracellular matrix proteins and fibrin. Immunohistochemical analysis revealed that fibrin deposition in sciatic nerve injury correlates spatially and temporally with nerve degeneration, whereas fibrin clearance correlates with nerve regeneration [23]. In multiple sclerosis, extensive accumulation of perivascular fibrin was observed in lesions. Fibrin deposits localized to inflamed blood vessels and in conjunction with increased immune cells infiltrated the area [24]. The probable mechanisms include its regulation of leukocyte-endothelial cell interactions and mediation of the expression of proinflammatory cytokines and chemokines [25, 26]. In addition, fibrinogen could induce activation of macrophage cells. Further studies of experimental autoimmune encephalomyelitis (EAE) proved that fibrinogen depletion by the use of ancrod decreased fibrin deposition and paralysis [21].

Inflammation evoked by an autoimmune reaction is a central event of the disease. Many studies have described in detail how the inflammatory process is initiated and sustained; it comprises complicated interactions between autoaggressive cells, costimulatory molecules, cytokines, chemokines and metalloproteinases (MMPs) $[1,27,28]$. We hypothesized that fibrinogen plays a role in IPM and fibrinogen depletion would be a beneficial therapy target. However, few reports have mentioned the role of fibrin(ogen) in IPM. Against our expectations, our results showed that defibrase-treated animals displayed more severe inflammation and necrosis, which were confirmed by histological examination.

What did this result imply and how did defibrase affect EAM? Defibrase is a thrombin-like proteinase which can cleave fibrinogen, which has a poorer linking ability than the fibrin polymer, and is therefore rapidly eliminated from the circulation by the reticuloendothelial cell system and/ or by secondary fibrinolysis. Fibrinogen is widely used in treatment of stroke, deep-vein thrombosis, peripheral arterial occlusion, and microcirculatory dysfunction. When we used defibrase in an EAM animal model, the result was opposite to our expectation in that defibrase-treated animals acquired more severe inflammation and concomitant fiber degeneration and necrosis.

We hypothesize that fibrin(ogen) probably has complex functions in inflammatory myopathy: On one hand, during the early period it leaks into in- flammatory tissues and is converted into soluble fibrin by thrombin. Then with the action of factor XIIla, soluble fibrin monomers cross link to form a stable fibrin molecular 'net'. In this fibrin-rich matrix, foreign and auto-antigens are captured and detained at local regions, which facilitates mononuclear cells to attack and eventually eliminate invaders. Meanwhile, fibrin and its degradation products also have a functional role at this stage by increasing vascular permeability and inducing expression of cytokines and chemotaxis.

On the other hand, during the late period of inflammation, persistent and excessive fibrin deposition hinders extracellular matrix remodeling and tissue repair. As a major matrix component within inflammatory areas, the fibrin net may represent a barrier between affected vessels and muscles and have a protective role in the early inflammatory process. Based on this hypothesis, we speculated that fibrinogen depletion reduced fibrin deposits in inflammatory muscle tissues and thus restricted fibrin matrix formation. This needs further study and data to validate.

Another mechanism of defibrase in EAM may be correlated with fibrinolysis and extracellular matrix remodeling. As mentioned above, IPM is characterized by the presence of inflammatory cells invading muscle tissue. Evidence showed that defibrase could induce release of plasminogen activator (PA) from endothelial cells and indirectly restrain plasminogen activator inhibitor (PAI) activity. UPA was identified as a critical component of the mammalian skeletal muscle regeneration process, for it prevents intramuscular fibrin accumulation and contributes to the adequate inflammatory response after injury [29]. It is also an important physiological activator in vivo of MMPs, which participate actively in the remodeling of tissues [30]. Studies demonstrated overexpression of MMPs (MMP2 and MMP9) on non-necrotic fibers, atrophic myofibers and invading $T$ lymphocytes in PM [31, 32]. Our previous studies (not published) demonstrated that immunostaining of both UPA and MMP9 was positive in muscle fibers of EAM animals, but was negative or feeble in normal muscles of controls. UPA and MMP9 antigens localized predominantly on the wall of small vessels, infiltrated mononuclear cells and affected muscle fibers. This distribution indicated the role of UPA and MMP9 in EAM, where degradation of the extracellular matrix occurred during inflammation, which facilitated migration of inflammatory cells across vascular endothelial cells for autoantigen-expressing myofibers. Taken together, these data suggested that as an intervention, the administration of defibrase would probably accelerate destruction of the extracellular matrix by activation of UPA, which would subsequently activate MMPs. 
In conclusion, our study demonstrates that defibrase fulfills an adverse role in EAM. Defibrase can serve as a favorable adjuvant in an animal model for human IPM, and defibrase-treated models could provide us more severe inflammation of myositis for therapeutic research use.

\section{Conflict of interests}

The authors declare no conflict of interests.

\section{References}

1. Dalakas MC, Hohlfeld R. Polymyositis and dermatomyositis. Lancet 2003; 362: 971-82.

2. Matsubara S, Takamori M. Experimental allergic myositis: strain 13 guinea pig immunised with rabbit myosin B fraction. Acta Neuropathol 1987; 74: 158-62.

3. Smith PD, Partridge TA. Macrophage migration inhibition studies of lymphocytes taken from guinea-pigs suffering from experimental polymyositis. Clin Exp Immunol 1976; 25: 133-8.

4. Momeni M, Carlier C, Baele P, et al. Fibrinogen concentration significantly decreases after on-pump versus off-pump coronary artery bypass surgery: a systematic point-of-care ROTEM analysis. J Cardiothor Vasc Anesth 2013; 27: 5-11.

5. Collins J, van Pijkeren JP, Svensson L, et al. Fibrinogen-binding and platelet-aggregation activities of a Lactobacillus salivarius septicaemia isolate are mediated by a novel fibrinogen-binding protein. Mol Microbiol 2012; 85: 862-77.

6. Ma A, Pan X, Xing Y, Wu M, Wang Y, Ma C. Elevation of serum CXCL16 level correlates well with atherosclerotic ischemic stroke. Arch Med Sci 2014; 10: 47-52.

7. Profumo A, Turci M, Damonte G, et al. Kinetics of fibrinopeptide release by thrombin as a function of $\mathrm{CaCl} 2$ concentration: different susceptibility of FPA and FPB and evidence for a fibrinogen isoform-specific effect at physiological Ca2+ concentration. Biochemistry 2003; 42: 12335-48.

8. Fibrinogen Studies C, Danesh J, Lewington S, et al. Plasma fibrinogen level and the risk of major cardiovascular diseases and nonvascular mortality: an individual participant meta-analysis. JAMA 2005; 294: 1799-809.

9. Jensen T, Kierulf P, Sandset PM, et al. Fibrinogen and fibrin induce synthesis of proinflammatory cytokines from isolated peripheral blood mononuclear cells. Thrombosis Haemostasis 2007; 97: 822-9.

10. Polterauer S, Grimm C, Seebacher V, et al. Plasma fibrinogen levels and prognosis in patients with ovarian cancer: a multicenter study. Oncologist 2009; 14: 979-85.

11. Gur-Wahnon D, Mizrachi T, Maaravi-Pinto FY, et al. The plasminogen activator system: involvement in central nervous system inflammation and a potential site for therapeutic intervention. J Neuroinflam 2013; 10: 124.

12. Puz P, Lasek-Bal A, Ziaja D, Kazibutowska Z, Ziaja K. Inflammatory markers in patients with internal carotid artery stenosis. Arch Med Sci 2013; 9: 254-60.

13. Kojima T, Tanuma N, Aikawa Y, Shin T, Sasaki A, Matsumoto $Y$. Myosin-induced autoimmune polymyositis in the rat. J Neurol Sci 1997; 151: 141-8.

14. Allenbach Y, Solly S, Gregoire S, et al. Role of regulatory $T$ cells in a new mouse model of experimental autoimmune myositis. Am J Pathol 2009; 174: 989-98.
15. Katsumata Y, Harigai M, Sugiura T, et al. Attenuation of experimental autoimmune myositis by blocking ICOSICOS ligand interaction. J Immunol 2007; 179: 3772-9.

16. Nakano J, Yoshimura T, Okita M, et al. Laminin-induced autoimmune myositis in rats. J Neuropathol Exp Neurol 2005; 64: 790-6.

17. Wang H, Schultz K, Elias K, Stachowski M, Loose C. Anti-infection trauma devices with drug-releasing and non-Fouling surface modification. J Orthop Trauma 2014; 28 Suppl. 1: S28-31.

18. Cruz-Topete D, Iwaki T, Ploplis VA, Castellino FJ. Delayed inflammatory responses to endotoxin in fibrinogen-deficient mice. J Pathol 2006; 210: 325-33.

19. Malyszko J, Bachorzewska-Gajewska H, Malyszko J, Iaina-Levin N, Kobus G, Dobrzycki S. Markers of kidney function in the elderly in relation to the new CKD-EPI formula for estimation of glomerular filtration rate. Arch Med Sci 2011; 7: 658-64.

20. Zhang W, Huang W, Jing F. Contribution of blood platelets to vascular pathology in Alzheimer's disease. J Blood Med 2013; 4: 141-7.

21. Akassoglou K, Adams RA, Bauer J, et al. Fibrin depletion decreases inflammation and delays the onset of demyelination in a tumor necrosis factor transgenic mouse model for multiple sclerosis. Proc Natl Acad Sci USA 2004; 101: 6698-703.

22. Gay FW. Early cellular events in multiple sclerosis. Intimations of an extrinsic myelinolytic antigen. Clin Neurol Neurosurg 2006; 108: 234-40.

23. Akassoglou K, Yu WM, Akpinar P, Strickland S. Fibrin inhibits peripheral nerve remyelination by regulating Schwann cell differentiation. Neuron 2002; 33: 861-75.

24. Wakefield AJ, More LJ, Difford J, McLaughlin JE. Immunohistochemical study of vascular injury in acute multiple sclerosis. J Clin Pathol 1994; 47: 129-33.

25. Altieri DC. Regulation of leukocyte-endothelium interaction by fibrinogen. Thromb Haemost 1999; 82: 781-6.

26. Smiley ST, King JA, Hancock WW. Fibrinogen stimulates macrophage chemokine secretion through toll-like receptor 4. J Immunol 2001; 167: 2887-94.

27. Tworek D, Kuna P, Mlynarski W, Gorski P, Pietras T, Antczak A. MIG (CXCL9), IP-10 (CXCL10) and I-TAC (CXCL11) concentrations after nasal allergen challenge in patients with allergic rhinitis. Arch Med Sci 2013; 9: 849-53.

28. Zwierzchowski TJ, Stasikowska-Kanicka O, Janus J, Konecki W, Danilewicz M, Fabis J. Assessment of apoptosis, MMP-1, MMP-3, TIMP-2 expression and mechanical and biochemical properties of fresh rabbit's medial meniscus stored two weeks under tissue culture conditions. Arch Med Sci 2014; 10: 167-73.

29. Lluis F, Roma J, Suelves M, et al. Urokinase-dependent plasminogen activation is required for efficient skeletal muscle regeneration in vivo. Blood 2001; 97: 1703-11.

30. Murphy G, Willenbrock F, Crabbe T, et al. Regulation of matrix metalloproteinase activity. Ann New York Acad Sci 1994; 732: 31-41.

31. Schoser BG, Blottner D, Stuerenburg HJ. Matrix metalloproteinases in inflammatory myopathies: enhanced immunoreactivity near atrophic myofibers. Acta Neurol Scand 2002; 105: 309-13.

32. Kieseier BC, Schneider C, Clements JM, et al. Expression of specific matrix metalloproteinases in inflammatory myopathies. Brain 2001; 124: 341-51. 